\title{
Is grand multiparity associated with an increased risk of dysglycaemia?
}

Received: 21 November 2005 / Accepted: 16 March 2006 / Published online: 11 May 2006

C) Springer-Verlag 2006

\begin{abstract}
Aims/hypothesis: We sought to determine the risk of diabetes and IGT/IFG with grand multiparity. Subjects, materials and methods: Women, aged $\geq 25$ years, from the Australian Diabetes, Obesity and Lifestyle Study and the Crossroads Undiagnosed Disease Study (a rural study in Victoria, Australia), participated in a household census (response 67 and $70 \%$, respectively), subsequently attending a biomedical examination that included an oral glucose tolerance test (58\% [6198] and 69\% [819]). Results: After adjusting for age, obesity and socio-economic status, diabetes, but not IGT/IFG, was less common among women with a parity of 1 to 2 (odds ratio $[\mathrm{OR}]=0.64[0.48-$ $0.84])$ and 3 to $4(\mathrm{OR}=0.72[0.53-0.96])$ than in grand multiparous women. This relationship was unrelated to past hysterectomy, use of the oral contraceptive pill or menopausal status. Conclusions/interpretation: Grand multiparity is associated with an increased risk of diabetes but not of IGT/IFG. We postulate that parity accelerates transition from IGT/IFG to diabetes, more than it does transition from normal glucose tolerance to IGT/IFG.
\end{abstract}

D. Simmons $(\triangle)$

University of Auckland Waikato Clinical School,

Waikato Hospital,

Private Bag 3200, Pembroke Street,

Hamilton, New Zealand

e-mail: simmonsd@waikatodhb.govt.nz

Tel.: +64-7-8398899

Fax: +64-7-8343615

D. Simmons

Department of Rural Health, University of Melbourne,

Shepparton, VIC, Australia

J. Shaw · A. J. Cameron · P. Zimmet

International Diabetes Institute,

Melbourne, VIC, Australia

A. McKenzie

Department of Rural Health, University of Melbourne,

Shepparton, VIC, Australia

S. Eaton

Northumbria Healthcare NHS Trust,

North Shields, UK
Keywords Diabetes $\cdot$ Hysterectomy $\cdot$ Menopause $\cdot$ Oral contraceptive pill $\cdot$ Parity

Abbreviations AusDiab: Australian Diabetes, Obesity and Lifestyle Study · CUDS: Crossroads Undiagnosed Disease Study · FPG: fasting plasma glucose - GDM: gestational diabetes - PG: plasma glucose ·

OR: odds ratio

\section{Introduction}

The relationship between parity and risk of diabetes remains unclear. After adjusting for obesity and age, some studies show increased risk of diabetes with increasing parity [1-3] and others show no relationship [4-8]. At least four studies have shown an increased risk of diabetes with nulliparity [3, 8-10], perhaps explaining why some studies have not shown a linear relationship. The relationship between risk of diabetes and parity may also vary with age, with one study showing more diabetes with grand multiparity among younger but not among older ( $>65$ years) women [3]; this may suggest that increased parity promotes earlier development of diabetes rather than increasing lifetime diabetes risk. Few studies have discussed the relationship between parity and either IGT or IFG, and those that did either found [2] or did not find a relationship $[5,7]$.

While most studies have adjusted for both age and weight, another potential confounder has been whether diabetes has been diagnosed or not [6]: the inclusion of undiagnosed diabetes prevents bias from the greater number of possible occasions when diabetes screening has occurred. Other potential confounders relate to the duration of fertility through either menopause (naturally or surgically) or the use of the oral contraceptive pill. The latter may have its own independent effect on the risk of diabetes with both increased [11,12] and reduced risk [13] having been reported.

We have now sought to determine whether diabetes and/ or IGT/IFG are independently related to grand multiparity 
(i.e. $\geq 5$ pregnancies lasting at least 6 months), after adjusting for potential confounders, in a large cohort of women who participated in either of two Australian population-based studies, the Australian Diabetes, Obesity and Lifestyle Study (AusDiab) [14, 15], and the Crossroads Undiagnosed Disease Study (CUDS) [16].

\section{Subjects, materials and methods}

\section{Study designs}

AusDiab used a stratified cluster sampling from 42 randomly selected Census Collector Districts across Australia between May 1999 and December 2000 as described elsewhere [15]. In AusDiab, districts containing fewer than 100 people aged $\geq 25$ years, those classified $100 \%$ rural, and those in which $10 \%$ or more of the population were Aboriginal or Torres Straits Islanders were excluded from the sample frame available for selection. CUDS involved randomly selected households across six small rural towns and from the regional centre in the Goulburn-Murray region in rural Victoria between June 2001 and March 2003 [17]. In CUDS, households were randomly selected for inclusion from residential address lists from local Shire Offices, stratified so that half were from the regional centre and one-twelfth from each of the six smaller towns. AusDiab and CUDS invited all usual residents within each selected household who were aged $\geq 25$ years to participate in a biomedical examination at a local survey centre. Both studies involved an initial census, during which information was collected and attendance at the biomedical examination negotiated. Identical questionnaires were used in each study, several of the staff worked on both studies, and the same laboratory was used for analysing samples. Householders not available at the initial interview were approached up to five times in AusDiab, and until contact was made (sometimes over ten times) in CUDS. Response rates to the initial census were $67 \%$ in AusDiab and $70 \%$ in CUDS. Of the women identified in the household surveys as being eligible, 58\% $(n=6198)$ and $69 \%(n=819)$, respectively, attended the biomedical examination.

\section{Survey procedures}

Subjects attended fasting between 07.00 and $10.00 \mathrm{~h}$. If fasting was reported to be $<8 \mathrm{~h}$, another appointment was made. Those treated with hypoglycaemic agents were requested not to take their medication on the day of the examination. An OGTT was undertaken, following World Health Organization guidelines, on all participants except those taking pharmacological hypoglycaemic medication and those who were pregnant [17]. Blood was collected into fluoride/oxalate tubes immediately before and $2 \mathrm{~h}$ after consumption (over $<5 \mathrm{~min}$ ) of a 300 -ml beverage containing $75 \mathrm{~g}$ glucose. Specimens were centrifuged, the plasma immediately separated and the glucose concentration measured using the glucose oxidase method and an automated analyser (AU600; Olympus Optical, Tokyo, Japan). Among subjects without known diabetes, those with fasting plasma glucose (FPG) of $\geq 7.0 \mathrm{mmol} / \mathrm{l}$ and/or 2-hour glucose (2-h PG) of $\geq 11.1 \mathrm{mmol} / 1$ were classified as having newly diagnosed diabetes mellitus; those with an FPG of $<7.0 \mathrm{mmol} / \mathrm{l}$ and $2-\mathrm{h} \mathrm{PG}$ of $7.8-11.0 \mathrm{mmol} / \mathrm{l}$ were classified as having IGT; and those with an FPG of 6.1$6.9 \mathrm{mmol} / \mathrm{l}$ and $2-\mathrm{h}$ PG of $<7.8 \mathrm{mmol} / 1$ were classified as having IFG.

The same questions regarding women's health were asked in both AusDiab and CUDS. These questions included: (1) Have you ever taken the oral contraceptive pill? (2) Have you had a hysterectomy; that is, an operation to remove the uterus? (3) Were the ovaries removed as well? And (4) How many pregnancies have you had that lasted at least 6 months ( 24 weeks)? Questions concerning the menopause were preceded by the statement: 'The next few questions are about menopause, or what some women refer to as the "change of life". Menopause is when periods have stopped for more than 6 months, and is often accompanied by symptoms such as hot flushes, irritability and palpitations.' The first question on the menopause was: have you gone through or are you now going through menopause? A number of older women answered 'no' or 'not sure' and hence women were considered menopausal or post-menopausal if they answered 'yes' directly, if they had had a hysterectomy and bilateral oophorectomy, or if they were aged over 55 years. Information given was not confirmed against medical records. Age at onset of menopause was taken as the self-reported age at which symptoms commenced. Socioeconomic status was assessed by the highest level of education received.

Height was measured to the nearest $0.5 \mathrm{~cm}$ without shoes using a stadiometer. Weight was measured to the nearest $0.1 \mathrm{~kg}$ using a mechanical beam balance after removal of shoes and excess clothing. BMI was calculated in kilogram per square metre and overweight defined as $25.0-29.9 \mathrm{~kg} / \mathrm{m}^{2}$ and obesity as $\geq 30.0 \mathrm{~kg} / \mathrm{m}^{2}$ [18]. These measurements were carried out according to the same protocols and equipment in both CUDS and AusDiab.

\section{Statistical analysis}

Data were analysed using SPSS 11.5 (SPSS, Chicago, IL, USA) and STATA 8.1 (Stata, College Station, TX, USA). Results shown are mean \pm standard deviation, median (range) or frequencies (percentages). Statistical significance was taken at the 5\% level and all tests are two-tailed. Discrete variables were compared using chi-squared test and continuous variables using analysis of variance. Parity has been grouped into para 0 , para 1-2, para 3-4 and para $5+$ based upon, and to allow comparison with our previous work [3]. Comparisons have been adjusted for age and BMI as continuous variables, and socio-economic status (post-secondary school qualification or not) by logistic regression and odds ratios (ORs) (95\% CIs shown). Adjustment for obesity was undertaken to ensure that any 
increase in diabetes risk with parity that was detected was not due to its relationship with both diabetes and parity. In view of the extent of association between age, BMI and parity, the logistic regression was also undertaken using age and BMI as categorical variables with similar findings. A case-control analysis was undertaken group-matching randomly selected para 1-2 women and para 3-4 women by 5 -year age group (twelve groups up to $80+$ years) and BMI (eight groups of $2 \mathrm{~kg} / \mathrm{m}^{2}$ from $<21$ to $33+\mathrm{kg} / \mathrm{m}^{2}$ ) with para $5+$, i.e. grand multiparous women, thereby creating three groups of identical size, BMI and age.

Adjustment for geographical clustering (defined as any of the areas in AusDiab or CUDS) was made using STATA. All the logistic regression analyses have been adjusted for clustering. Ethnicity was not readily collectable (besides Aboriginal and Torres Straits Islands participants, $n=90$ ), but 371 subjects $(5.3 \%)$ were not of European Australian descent. The studies were approved by the relevant ethics committees and signed individual consent was obtained.

\section{Results}

Figure 1 shows the numbers of participants by hysterectomy and menopausal status. Overall, 3,863 women were considered menopausal/postmenopausal and 2,919 women were considered premenopausal. The median (interquartile range) age at which menopausal symptoms commenced (among the $90.2 \%$ who reported an age) was 48 (44-50) years. Table 1 shows the crude mean age and BMI by history of hysterectomy, oral contraceptive pill use, parity, and menopausal status. Figure 2 shows the prevalence of diabetes (known and previously undiagnosed combined) by age and parity. Table 2 shows that after adjusting for age, obesity and socio-economic status, a history of hysterectomy was unrelated to glucose intolerance. Grand multiparous women were significantly more likely to have diabetes than women with a parity of either 1 to 2 or 3 to 4 and non-significantly more likely to have diabetes than nulliparous women. No significant difference between women with known and undiagnosed diabetes was found. The risk of IGT/IFG was not related to parity. The risk of any abnormal glucose tolerance was marginally higher among grand multiparous women. Repeating the analyses using the case-control approach gave a prevalence of diabetes by parity of $13.0 \%$ (para $1-2$ ), $10.8 \%$ (para 3-4) and $16.6 \%$ (para $5+)(p=0.018)$. Using this approach, grand multiparous women were $1.47(1.10-1.96)$ times as likely to have diabetes than para 1-4 women. Prevalence of IGT/IFG was comparable and overall dysglycaemia nonsignificantly higher (OR 1.22 [0.99-1.51]) among para 5+ women.
Fig. 1 Hysterectomy and menopausal status of women. ${ }^{1}$ Hysterectomy without known bilateral oophorectomy

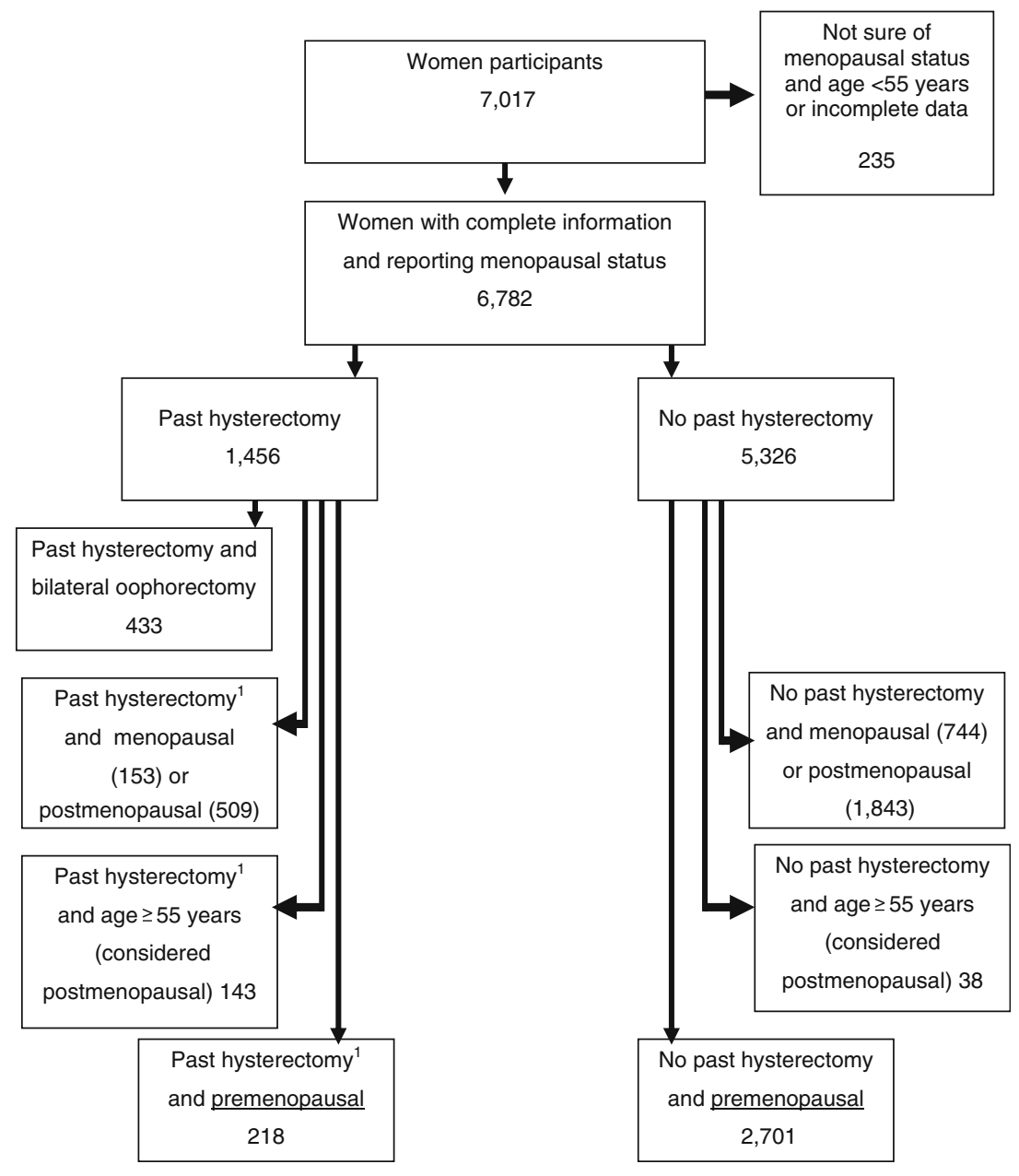


Table 1 Characteristics of study population according to hysterectomy status, oral contraceptive pill use, parity, menopausal status and past GDM

\begin{tabular}{lrll}
\hline & $n$ & Age (years) & BMI $\left(\mathrm{kg} / \mathrm{m}^{2}\right)$ \\
\hline Hysterectomy status & & & \\
No & 5,326 & $49 \pm 15^{* * *}$ & $26.6 \pm 5.6^{* * *}$ \\
Yes & 1,456 & $59 \pm 12$ & $27.9 \pm 5.6$ \\
OCP use & & & \\
No & 1,650 & $64 \pm 15$ & $27.1 \pm 5.4$ \\
Yes & 5,132 & $47 \pm 12^{* * *}$ & $26.8 \pm 5.7$ \\
If OCP use & & & \\
Past & 4,124 & $49 \pm 12$ & $27.0 \pm 5.8$ \\
Current & 1,008 & $41 \pm 12^{* * *}$ & $26.1 \pm 5.2 * * *$ \\
Parity & & & \\
0 & 973 & $45 \pm 16$ & $25.9 \pm 5.7$ \\
1-2 & 2,868 & $49 \pm 14$ & $26.7 \pm 5.7$ \\
3-4 & 2,392 & $54 \pm 13$ & $27.2 \pm 5.4$ \\
5+ & 549 & $63 \pm 12^{* * *}$ & $28.3 \pm 5.6^{* * *}$ \\
Menopause & & & \\
Past & 2,966 & $65 \pm 10$ & $27.5 \pm 5.3$ \\
Current & 897 & $51 \pm 5$ & $27.6 \pm 5.8$ \\
No & 2,919 & $38 \pm 7 * * *$ & $26.1 \pm 5.8^{* * *}$ \\
\hline
\end{tabular}

Data are $n$, means \pm SD

$O C P$ Oral contraceptive pill

$* * * p<0.001$ for yes vs no and other category comparisons

Being peri-menopausal was associated with an increased risk of having IGT/IFG, but not of having diabetes or any abnormal glucose tolerance. Age at onset of menopause was not associated with the risk of abnormal glucose tolerance. Use of the oral contraceptive pill was associated with a lower risk of any abnormal glucose tolerance overall. However, among women who had at any time used the oral contraceptive pill, IGT/IFG and dysglycaemia overall were more likely in current than in past users. The median estimated time for use of the oral contraceptive pill was 5 to 10 years, and there was no relationship between duration of use and glucose tolerance. Grand multiparous women were 1.75-fold (1.34-2.30) more likely to have taken the oral contraceptive pill than nulliparous women,

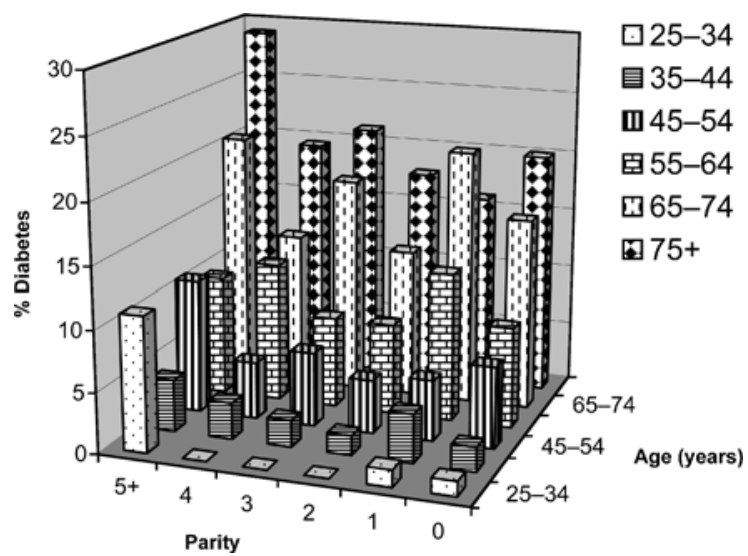

Fig. 2 Prevalence of diabetes by parity and age but 0.72 -fold (0.57-0.91) and 0.72-fold (0.57-0.90) less likely when compared with para 1-2 and para 3-4 women, respectively.

Table 3 shows the results of a multivariate logistic regression including parity, history of hysterectomy, history of oral contraceptive pill use, menopausal status with risk of diabetes, IGT/IFG or any abnormal glucose tolerance after adjusting for age, obesity, socio-economic status and cluster. Grand multiparity remained associated with a significantly greater risk of diabetes (vs para 1-4) in both analyses.

\section{Discussion}

In our study, there was clearly a significant relationship between grand multiparity and an increased risk of diabetes, diagnosed and previously undiagnosed, after adjusting for age, BMI and socio-economic status. Several previous studies of the relationship between parity and diabetes have been hampered either by smaller numbers or inclusion of nulliparous women in a regression analysis, or they failed to include an OGTT. Conversely, there was clearly no relationship between parity and risk of IGT/IFG. Such a relationship has been shown previously in postmenopausal white American women [2] but not across ages in Hispanic and non-Hispanic Americans [7], or in Melanesians, South Asians, Chinese or Creole participants in a range of studies [5].

We wonder whether grand multiparity hastens progression from IGT/IFG to diabetes, not only in some ethnic groups [19], but perhaps also in different age cohorts, and whether this is why such a factor with a relatively limited effect (i.e. grand multiparity) could have this differential effect between diabetes and IGT/IFG. This could mean that in the older age group, those who were to develop diabetes have largely done so (or died). Our study is, of course, cross-sectional, and a prospective study would be superior. There was a marginally increased risk of abnormal glucose tolerance overall, suggesting that the effect of parity is not entirely through increasing the risk of progression from IGT/IFG to diabetes, but also, to a lesser extent, through increasing the risk of progression from normal to abnormal glucose tolerance. Again, this relationship may be different if studied prospectively and could have been confounded by differential mortality.

Possible mechanisms for the increased risk of diabetes with grand multiparity are unclear, particularly without a major gradation of risk from para 1 women upwards. KritzSilverstein et al. showed a minor gradation in risk between para 2 and para 5 (comparable to that found here between para 1-2 and para 3-4), and then a major increase in para $6+$ women [2], and it may be that this represents some kind of threshold effect, above which harm occurs. Like other investigators, we adjusted for obesity, as grand multiparous women are also considerably more obese. While this seems an important adjustment, it is of course measured currently, i.e. in the present, while parity was established in the past. If the potential mechanisms linking parity with diabetes act 
Table 2 Adjusted ORs of having abnormal glucose tolerance according to hysterectomy status, oral contraceptive pill use, parity and menopause

\begin{tabular}{|c|c|c|c|}
\hline & Diabetes (known and newly diagnosed) & IGT/IFG & Any of DM/IGT/IFG \\
\hline \multicolumn{4}{|l|}{ Hysterectomy status } \\
\hline No & 1 & 1 & 1 \\
\hline Yes & $1.09(0.88-1.35)$ & $0.92(0.78-1.08)$ & $0.95(0.83-1.10)$ \\
\hline \multicolumn{4}{|l|}{ OCP use } \\
\hline No & 1 & 1 & 1 \\
\hline Yes (current/past) & $0.81(0.64-1.03)$ & $0.98(0.82-1.17)$ & $0.86(0.73-1.00)$ \\
\hline Current & $1.12(0.75-1.67)$ & $1.55(1.23-1.94)$ & $1.56(1.26-1.93)$ \\
\hline Past & 1 & 1 & 1 \\
\hline \multicolumn{4}{|l|}{ Parity } \\
\hline 0 & $0.79(0.53-1.16)$ & $0.94(0.69-1.28)$ & $0.84(0.64-1.11)$ \\
\hline $1-2$ & $0.66(0.49-0.90)$ & $1.05(0.82-1.34)$ & $0.84(0.68-1.05)$ \\
\hline $3-4$ & $0.73(0.55-0.98)$ & $0.98(0.78-1.27)$ & $0.83(0.67-1.03)$ \\
\hline $5+$ & 1 & 1 & 1 \\
\hline \multicolumn{4}{|l|}{ Menopause } \\
\hline Past & 1 & 1 & 1 \\
\hline Current & $0.83(0.54-1.26)$ & $1.33(1.05-1.69)$ & $1.09(0.87-1.36)$ \\
\hline No & $1.16(0.76-1.17)$ & $1.08(0.83-1.41)$ & $1.00(0.79-1.29)$ \\
\hline
\end{tabular}

ORs (95\% CIs) are adjusted for age and BMI (continuous variables), socioeconomic status and cluster

Parity comparison has $5+$ as referent to compare each parity group with grand multiparity

$D M$ Diabetes mellitus, $O C P$ oral contraceptive pill

through obesity (at least partially), adjustment may have removed much of its impact. We cannot see any other way that grand multiparity could influence insulin resistance beyond delivery, but could imagine some permanent marginal effect on beta cell mass, similar to that which occurs with multiple episodes of gestational diabetes, which predispose to earlier type 2 diabetes [20]. This presumably affects those at high risk anyway, increasing the risk in later years (as shown here), but perhaps also influencing a number of individuals who would otherwise have been normal.

Although data relating to gestational diabetes (GDM) were collected, these have not been included, as there are multiple confounders in such cross-sectional data, particularly the validity of self-reporting of past GDM and of screening for GDM, the changing penetration of screening for GDM, changing and differing criteria for GDM across Australia over the last 75 years, and the changing prevalence of obesity and the metabolic syndrome. The diagnosis of GDM, more likely with increasing parity, could have been associated with a greater proportion of subjects diagnosed as diabetic, but numbers were too small to assess this. We hope that at some point prospective data will become available to test the importance of past GDM on the relationship between parity and diabetes risk.

Many large surveys such as these have limited participation and there is always a potential bias from self-selection. This is a survivor cohort with unpredictable associated biases. Our definition of menopause could have confounded the sub-analyses. Most menopausal women were able to provide an age at which symptoms commenced. However, some older women indicated they were unaware of when the menopause commenced. We believe a cut-off of 55 years is reasonable. We included past hysterectomy and previous oral contraceptive pill use, because of their influence on parity. In the end, neither had an effect on the parity analyses. Past hysterectomy was, as expected, unrelated to the risk of diabetes. However,

Table 3 Logistic regressions of parity, oral contraceptive pill use, hysterectomy and menopause with dysglycaemia after adjusting for confounders

\begin{tabular}{llll}
\hline & Diabetes (known and newly diagnosed) & IGT/IFG & Any of DM/IGT/IFG \\
\hline Hysterectomy vs no & $1.08(0.87-1.34)$ & $0.90(0.76-1.06)$ & $0.94(0.82-1.09)$ \\
OCP use vs no & $0.81(0.64-1.03)$ & $0.94(0.79-1.13)$ & $0.85(0.72-0.99)$ \\
Parity 0 vs 5+ & $0.76(0.51-1.14)$ & $0.93(0.67-1.27)$ & $0.82(0.62-1.08)$ \\
$1-2$ vs 5+ & $0.67(0.49-0.91)$ & $1.04(0.82-1.34)$ & $0.84(0.68-1.05)$ \\
3-4 vs 5+ & $0.74(0.55-0.99)$ & $0.99(0.78-1.26)$ & $0.83(0.67-1.04)$ \\
Menopause & $1.10(0.75-1.62)$ & $1.30(1.03-1.65)$ & $1.15(0.92-1.42)$ \\
\hline
\end{tabular}

ORs (95\% CIs) are adjusted for age, obesity (obese, overweight or normal), socioeconomic status and cluster

$D M$ Diabetes mellitus, $O C P$ oral contraceptive pill 
current, but not past use of the oral contraceptive pill was associated with a higher risk of any abnormality in glucose tolerance. There are clearly a great number of confounders in what type of women take the oral contraceptive pill, the duration taken, and whether a subject is a current user or not, and prospective studies are better suited to find this out. Indeed, prior prospective studies using either a highdose [11] or low-dose [12] oral contraceptive pill have shown an increased risk of diabetes, although a recent study showed that risk of diabetes was reduced with combined oestrogen and progestin [13]. We did not enquire about the possibility of subjects having polycystic ovarian syndrome, and those with insulin resistance have had worsening of glucose tolerance with the oral contraceptive pill [21].

In summary, we have shown in a large Australian cohort of women of predominantly European descent that grand multiparity was associated with an increased risk of diabetes and of abnormal glucose tolerance overall, but not of IFG/IGT. We postulate that grand multiparity accelerates transition through IGT/IFG and, to a lesser degree, increases transition from normal glucose tolerance to IGT/IFG. Prospective studies are needed to confirm these findings.

Acknowledgements We thank the Crossroads team for their work and the local communities for their support. The Crossroads Undiagnosed Disease Study was funded by the International Diabetes Institute and the University of Melbourne. The Department of Rural Health is funded under the Department of Health and Ageing Rural Health programme. We thank G. Joshy for statistical assistance. The AusDiab team are grateful to the following for their support of the study: the Commonwealth Dept of Health and Aged Care, Abbott Australasia, Alphapharm, Aventis Pharmaceutical, AstraZeneca, Bristol-Myers Squibb Pharmaceuticals, Eli Lilly (Aust), GlaxoSmithKline, Janssen-Cilag (Aust), Merck Lipha, Merck Sharp \& Dohme (Aust), Novartis Pharmaceutical (Aust), Novo Nordisk Pharmaceutical, Pharmacia and Upjohn, Pfizer, Roche Diagnostics, Sanofi Synthelabo (Aust), Servier Laboratories (Aust), BioRad Laboratories, HITECH Pathology, the Australian Kidney Foundation, Diabetes Australia, Diabetes Australia (Northern Territory), Queensland Health, South Australian Department of Human Services, Tasmanian Department of Health and Human Services, Territory Health Services, Victorian Department of Human Services and Health Department of Western Australia. We are also enormously grateful to A. Allman, M. Dalton, D. Dunstan, A. Meehan, C. Reid, A. Stewart, R. Tapp and F.Wilson for their invaluable contribution to the field activities of AusDiab.

\section{References}

1. O'Sullivan JB, Gordon T (1966) Childbearing and diabetes mellitus. Publication no. 1000, ser. 11, no. 21, National Center for Health Statistics, Vital and Health Statistics, Washington

2. Kritz-Silverstein D, Barrett-Connor E, Wingard DL (1989) The effect of parity on the later development of non-insulin dependent diabetes mellitus or impaired glucose tolerance. N Engl J Med 321:1214-1219

3. Simmons D (1992) Parity, ethnic group and the prevalence of non-insulin dependent diabetes. The Coventry Diabetes Study. Diab Med 9:706-709
4. Boyko EJ, Alderman BW, Keane EM, Baron AE (1990) Effects of childbearing on glucose tolerance and NIDDM prevalence. Diabetes Care 13:848-854

5. Collins VR, Dowse GK, Zimmet PZ (1991) Evidence against association between parity and NIDDM from five population groups. Diabetes Care 14:975-981

6. Manson JE, Rimm EB, Colditz GA et al (1992) Parity and incidence of non-insulin dependent diabetes. Am J Med 93: 1318

7. Alderman BW, Marshall JA, Boyko EJ, Markham KA, Baxter J, Hamman RF (1993) Reproductive history, glucose tolerance and NIDDM in Hispanic and non-Hispanic white women. Diabetes Care 16:1557-1564

8. Cowan LD, Go OT, Howard BV, Devereux RB, Pettitt DJ, Fabsitz RR et al (1997) Parity, postmenopausal estrogen use and cardiovascular disease risk factors in American Indian Women: the Strong Heart Study. J Womens Health 6:441-449

9. Charles MA, Pettitt DJ, McCance DR, Hanson RL, Bennett PH, Knowler WC (1994) Gravidity, obesity and non-insulin dependent diabetes among Pima Indian women. Am J Med 97:250-255

10. Hanley JG, McKeown-Eyssen G, Harris SB et al (2002) Association of parity with risk of type 2 diabetes and related metabolic disorders. Diabetes Care 25:690-695

11. Chasan-Taber L, Willett WC, Stampfer MJ et al (1997) A prospective study of oral contraceptives and NIDDM among US women. Diabetes Care 20:330-335

12. Rimm EB, Manson JE, Stampfer MJ et al (1992) Oral contraceptive use and the risk of type 2 (non-insulin dependent) diabetes mellitus in a large prospective study of women. Diabetologia 35:967-972

13. Margolis KL, Bonds DE, Rodabough RJ et al (2004) Effect of oestrogen plus progestin on the incidence of diabetes in postmenopausal women: results from the Women's Health Initiative Hormone Trial. Diabetologia 47:1175-1187

14. Dunstan DW, Zimmet PZ, Welborn TA et al (2002) The rising prevalence of diabetes and impaired glucose tolerance: the Australian Diabetes, Obesity and Lifestyle Study. Diabetes Care 25:829-834

15. Dunstan D, Zimmet P, Welbourn T, Cameron A, Shaw J, de Courten M (2002) The Australian diabetes, obesity and lifestyle study (AusDiab) methods and response rates. Diabetes Res Clin Pract 57:119-129

16. Simmons D, McKenzie A, Eaton S et al (2005) Choice and availability of take-away and restaurant food is not related to the prevalence of adult obesity in rural communities in Australia. Int J Obesity Lond 703-710

17. World Health Organization (1999) Definition, diagnosis and classification of diabetes mellitus and its complications: report of a WHO consultation. Part 1. Diagnosis and classification of diabetes mellitus, WHO, Geneva

18. World Health Organization (1998) Obesity-preventing and managing the global epidemic: report of a WHO consultation on obesity. WHO, Geneva

19. Unwin N, Shaw J, Zimmet P, Alberti G (2002) International Diabetes Federation IGT/IFG consensus statement. Report of an Expert Consensus Workshop 1-4 August 2001, Stoke Poges, UK. Diab Med 19:708-723

20. Peters RK, Kjos SL, Xiang A, Buchanan TA (1996) Long-term diabetogenic effect of single pregnancy in women with previous gestational diabetes. Lancet 347:227-230

21. Diamanti-Kandarkis E, Baillargeon JP, Iuorno MJ, Jakubowicz DJ, Nestler JE (2003) A modern medical quandary: polycystic ovary syndrome, insulin resistance, and oral contraceptive pills. J Clin Endocrinol Metab 88:1927-1932 